\title{
Criminologie
}

\section{Analyse spatiale et criminologie}

\section{Daniel Élie}

Volume 27, numéro 1, 1994

Analyse spatiale du crime

URI : https://id.erudit.org/iderudit/017345ar

DOI : https://doi.org/10.7202/017345ar

Aller au sommaire du numéro

Éditeur(s)

Les Presses de l'Université de Montréal

ISSN

0316-0041 (imprimé)

1492-1367 (numérique)

Découvrir la revue

Citer cet article

Élie, D. (1994). Analyse spatiale et criminologie. Criminologie, 27(1), 7-21. https://doi.org/10.7202/017345ar

\section{Résumé de l'article}

This article addresses the spatial analysis of crime. Following a brief historical overview, the author discusses major conceptual and methodological issues relating to the study of space and crime. Emphasis is placed on the description of spatial statistical tools (i.e. spatial mean, standard distance, turbulence...) and on the problem of spatial autocorrection. Two maps are presented and discussed. d'utilisation que vous pouvez consulter en ligne.

https://apropos.erudit.org/fr/usagers/politique-dutilisation/ 
This article addresses the spatial analysis of crime. Following a brief historical overview, the author discusses major conceptual and methodological issues relating to the study of space and crime. Emphasis is placed on the description of spatial statistical tools (i.e. spatial mean, standard distance, turbulence...) and on the problem of spatial autocorrection. Two maps are presented and discussed.

L'intention de ce texte est de dresser une esquisse des rôles et possibilités des analyses spatiales en criminologie. Dans ce contexte, il faut comprendre par analyse spatiale l'ensemble des méthodes qualitatives et quantitatives présentes dans les démarches désignées généralement comme une écologie ou encore géographie de la criminalité. Après un bref historique et une suggestion de bilan, nous examinerons les utilités et apports de l'analyse spatiale, puis les moyens actuellement disponibles dans ce domaine. Nous soumettons également deux exemples contrastés tirés de nos travaux. En conclusion, nous présentons quelques concepts ou notions qui peuvent accompagner utilement cette approche.

\section{HISTORIQUE}

Les distributions spatiales de la criminalité sont observées et interprétées depuis fort longtemps. Il s'agit d'une longue tradition dont l'origine débute en même temps que la criminologie. De façon plus précise, on peut considérer que ces types de données ont été recueillies et utilisées dès l'élaboration des premières statistiques sur la criminalité au début du $19^{\mathrm{e}}$ siècle en Europe. Guerry (1833), Quételet (1835) utilisaient déjà des méthodes d'illustration géographique. Également, Mayhew (1862) analysait la criminalité et les problèmes sociaux en Angleterre et au pays de Galles en utilisant des méthodes similaires. Donc, l'examen des répartitions spatiales du crime a suscité l'intérêt des chercheurs en criminologie dès le début de cette discipline.

Histoire ancienne, mais aussi histoire souvent interrompue. Nous avons pu observer ce fait en dressant un inventaire et une tentative de bilan des études faites et présentées de 1832 à 1982 (voir Élie et Théorêt 1984).

1. Professeur, École de criminologie, Université de Montréal, C.P. 6128, Montréal, $\mathrm{H} 3 \mathrm{C} 3 \mathrm{~J} 7$. 
Nous avons repéré 71 travaux publiés au cours de cette période. On constate toutefois que de 1864 (Mayhew) à 1929 (Shaw et McKay) peu, sinon aucune, recherche importante n'a laissé de traces très visibles. L'examen de la spatialité a ainsi été pratiquement abandonné durant une soixantaine d'années. Il a connu un autre recul peu après la seconde guerre mondiale. Il s'agissait toutefois d'un arrêt moins prononcé que le précédent. En effet, Baldwin (1975), dans une tentative d'évaluation de l'approche écologique et géographique, signale au moins vingt études majeures en GrandeBretagne durant cette période.

On constate, par ailleurs, que les concepts utilisés dans l'ensemble des travaux sont liés aux approches théoriques dominantes en criminologie selon les époques. $\grave{A}$ ce point, il faut mentionner notamment l'anomie, l'urbanisation, l'urbanité, la pauvreté, etc. Il semble que l'approche écologique structurale ait été adoptée dans la majorité des études examinées. En effet, elles postulent presque toutes un déterminisme de la culture, de la classe, du statut social ou de façon générale de la structure sociale dans leur relation avec la criminalité.

Il est peut-être intéressant de considérer, à cette étape et en guise de bilan. les principales critiques adressées à l'approche écologique ou géographique en criminologie. Elles sont nombreuses et relativement sévères. Il semble impossible de les présenter de manière détaillée et exhaustive dans le cadre restreint de ce texte. Toutefois, en procédant par recoupement parmi les argumentations et les opinions d'auteurs, on peut déceler quatre remarques importantes formulées au sujet de cette démarche de recherche.

En premier lieu, on a souvent interrogé la valeur des données. Qu'estce qui est cartographié ? La question sur ce point désigne avec un soupçon soutenu les statistiques et informations officielles au sujet de la criminalité. On soutient que toute recherche dans laquelle on utilise ces données relève du fakirisme parce qu'elles ne représentent pas la criminalité. On souligne qu'on est en fait amené à cartographier les intérêts, perceptions et réactions des agences de contrôle social et de ceux qui les manipulent. Ce point de vue étant assez connu (et protubérant), il ne semble pas nécessaire d'élaborer.

On évoque également le sophisme écologique (ecological fallacy) dans le sens indiqué par Robinson. On reproche à ces études de lier la criminalité aux caractéristiques d'un groupe d'habitants d'une zone et même d'attribuer ces caractéristiques aux délinquants. On déplore tout spécialement la confusion entre corrélations écologiques et corrélations individuelles. Dans ce point de vue, les méthodes statistiques utilisées sont donc réputées fallacieuses.

Par ailleurs, d'autres critiques sont adressées aux types de crimes analysés. En effet, la dépendance vis-à-vis des données officielles contraint souvent les chercheurs à se limiter à un nombre restreint de types de crimes, 
tels que le cambriolage. l'homicide, le vol d'auto, ou le vol à main armée. Cette restriction est signalée comme peu payante pour le développement théorique dans le domaine.

Enfin, tout ceci étant posé, certains admettent que la prise en compte de l'aspect spatial, de la répartition géographique des événements, puisse constituer un ajout ou complément intéressant, sans plus, à d'autres genres de recherche. Ainsi, par exemple, l'examen de la concentration ou de la dispersion d'un type d'incident pourrait préciser le sens des taux ou des moyennes. Celui des déplacements également pourrait amener des informations instructives. L'intérêt serait de cet ordre.

Ces commentaires semblent judicieux. Mais ils ne peuvent évacuer des questions importantes, liées à la spatialité. et qui demeurent incontournables dans toute réflexion éclairée et pertinente au sujet de l'évolution de plusieurs types de crime. Il est permis de penser que cette évolution spatiale n'est pas nécessairement et toujours aléatoire. Ce point est peut-être le plus important à considérer. Quelqu'un a fait remarquer que l'incertitude est beaucoup plus confortable lorsqu'on en est certain. Or, chaque fois qu'on doit écarter l'idée que le hasard seul explique une configuration ou un déplacement spatial des crimes, on est amené du même coup à envisager la possibilitê d'un savoir. Autrement dit, les configurations observées dans ces cas se prêtent à une compréhension. En ce sens, il semble tout à fait raisonnable d'admettre, dans certains cas. l'existence de facteurs environnementaux actifs dont il reste à découvrir la nature et les modes d'influence. Au bout du compte, et à tout prendre, les commentaires rencontrés laissent intact cet aspect du sujet et soulignent seulement les difficultés d'élaboration de ce savoir.

Depuis une douzaine d'années on observe un regain d'intérêt pour cette approche, et ceci se produit dans un contexte de développements technologiques importants, notamment dans le domaine de l'informatique. Cette conjoncture semble prometteuse d'enrichissements pour la criminologie et pour les pratiques méthodologiques.

Ce regain d'intérêt se manifeste dans l'ambiance par la présence de plusieurs textes ou regroupement de textes remarquables. Pour n'en citer que quelques-uns, on peut mentionner: Brantingham et Brantingham (1981): Figlio, Hakim et Rengert (1986); Georges-Abeyie et Harries (1980) : Byrne et Sampson (1986); Maltz, Gordon et Friedman (1991); Reiss et Tonry (1986).

Les contenus des travaux sus-mentionnés mettent en relief les progrès accomplis dans ce domaine et signalent les moyens employés dans ce type de démarche ainsi que ses fonctions et utilités. Nous considérons d'abord ce dernier point. 
En premier lieu, on trouve la possibilité d'améliorer l'interprétation des indices et des taux de criminalité. En effet, l'expression du nombre de crimes en taux par 1000 ou 100000 habitants constitue une mesure très rudimentaire et contient de nombreux pièges. Des aspects importants du phénomène peuvent demeurer occultés à cause de l'utilisation d'une telle mesure. Or, l'analyse de la répartition géographique devient un complément obligeant dans de nombreux cas. Ainsi, une augmentation du taux de cambriolage peut se produire avec des configurations spatiales de plusieurs types. Dans un cas, on peut observer une intensification des incidents dans une zone très limitée. À l'opposé, il est possible qu'elle s'accompagne d'un étalement rapide sur tout un territoire. Selon la configuration, le même nombre peut présenter des significations très différentes. Dans cet exemple, le deuxième cas, i.e. accroissement avec étalement, signale une situation sociale plus inquiétante. On peut également remarquer qu'un taux constant dans un même territoire durant une certaine période n'exprime pas des modifications éventuelles de la configuration spatiale dans ce territoire. Or, cette donnée peut être de grande importance dans plusieurs cas de recherche. On est ainsi amené à penser que l'analyse spatiale devrait être utilisée de manière plus courante à cause du type d'information qu'elle fournit par elle-même et aussi parce qu'elle aide à transformer les statistiques sociales en information véritable.

Par ailleurs, on doit noter que l'étalement géographique d'un phénomène peut se mesurer par l'accroissement des taux autour d'une zone centrale. Mais très souvent l'usage des taux par secteurs ou zones aussi désirable soit-il contraint à une cartographie par «plage » qui peut occulter des configurations intéressantes. Des informations sont ainsi perdues que le raffinement de l'analyse par cartographie ponctuelle, évoquée ici, retient facilement, surtout lorsque la question de recherche est posée en micromilieu.

Cette approche peut également conduire à des interprétations plus fines des coefficients de corrélation et de régression. On sait depuis quelque temps que la corrélation observée entre l'évolution d'un fait social quelconque et celle d'un type de crime peut varier considérablement selon le niveau d'agrégation spatiale. Ainsi. une valeur obtenue dans un micro-milieu (exemple, un quartier), ne sera pas nécessairement trouvée dans le macromilieu (exemple. la ville autour du quartier). Le fait est déroutant et s'oppose aux généralisations hâtives. Tout chercheur ou lecteur avisé doit retenir la question : une corrélation où?

Mais l'analyse spatiale peut apporter davantage à ce chapitre et une recherche récente illustre bien le sujet. On étudiait la relation entre la présence de points de vente de drogues dans un district policier de Montréal et l'évolution des cambriolages et des vols à main armée. L'analyse a révélé 
une absence de proximité entre les trois faits. Ainsi, la corrélation antérieurement déclarée doit être interprétée de manière différente.

Il semble opportun de signaler une autre application intéressante de l'approche spatiale. Il s'agit de la construction des cartes dites «cognitives ». On étudie constamment l'évolution spatio-temporelle des crimes, mais on se préoccupe également des représentations mentales des citoyens à ce sujet. de leurs perceptions et surtout de la peur du crime. Ce sentiment détermine un bon nombre de comportements chez les citadins et entretient un véritable marché de l'angoisse. Dans ce domaine, les élaborations de cartes «mentales» se sont avérées très utiles et instructives. Un bon exemple de ce type d'étude se trouve dans un texte de Pyle (1980). Plus avant dans cet article on soumet un autre type d'application de la méthode au cours d'une analyse de la peur du crime dans le quartier Côte-des-Neiges à Montréal.

Il faut également signaler à ce sujet 1'analyse de la peur du crime faite par Tremblay, Cordeau et Kaczorowski (1990) dans un quartier du centreville de Montréal.

Par ailleurs, la mise à l'épreuve de plusieurs théories criminologiques implique l'examen des mouvements spatio-temporels des événements et des acteurs. Il en est ainsi, par exemple, de la théorie de Felson et Cohen, telle que testée par Sherman, Gartin et Buerger (1989). Dans le même ordre d'idées, celui des vérifications d'hypothèses, il est tout à fait possible, en utilisant les mêmes types de méthodes descriptives, de procéder à certaines recherches évaluatives en utilisant surtout l'examen des déplacements spatiaux de la criminalité. Ce point intéresse surtout l'implantation des politiques de prévention du crime.

En dernier lieu, il faut signaler un aspect des caractéristiques distributionnelles qui a rencontré jusqu'à maintenant peu d'attention de la part des chercheurs et analystes en criminologie, malgré sa grande importance. 11 s'agit de l'autocorrélation spatiale. Une présentation en sera faite plus avant.

Le sujet a été abordé par Costanzo, Halperin et Gale (1986), également par Élie et Legendre (1992). Le traitement de cet aspect de la spatialité a des implications méthodologiques assez sérieuses. On peut, sur ce point, souligner deux faits exemplaires et lourds de conséquences.

La présence d'autocorrélation positive signifie que les faits se répartissent sur une aire par zones et gradients d'intensités homogènes et qu'ils ne sont pas indépendants les uns des autres. Or, lorsqu'on prélève un échantillon sur une surface, on postule que chaque observation constitue une information nouvelle. La présence de l'autocorrélation fausse cette procédure puisqu'il y a redondance dans les données. Ceci a des implications vis-à-vis 
du choix d'une distribution d'échantillonnage appropriée. De plus, l'expérience prise dans les autres disciplines montre que la présence d'autocorrélation influence les résultats des tests statistiques courants, les rendant peu fiables (on rejette plus facilement l'hypothèse de différence nulle) (voir Élie et Legendre, op. cit.). Cette nouvelle donnée porte un éclairage différent sur les tests multivariés employés en criminologie et la pertinence de leur utilisation.

Nous évoquons plus avant des méthodes de détection de l'autocorrélation. Il serait certainement opportun d'examiner en même temps, de manière formelle et satisfaisante, les causes du phénomène. Nous considérons toutefois que cette réflexion dépasse le cadre de cet article. Il s'agit en effet d'une question assez vaste et d'une question de recherche relativement nouvelle. Tout en soulignant qu'il n'existe pas encore sur ce sujet, et dans notre domaine, de connaissances certaines, on peut émettre cependant plusieurs hypothèses conjecturales. Nous en retenons deux principales, pour faire bref.

Premièrement, on peut supposer que les causes du phénomène peuvent varier selon les types d'incidents qui intéressent la criminologie, selon la période, la zone et la composition démographique. On entrevoit aisément la complexité des combinaisons possibles.

Deuxièmement, et s'il est possible d'évoquer une dynamique ou un mouvement général dans notre domaine, il est utile de retenir d'abord les comportements d'évitement de la part des victimes potentielles de différents types de crimes. Ces évitements (i.e. protections ou mesures de prévention) détermineraient, dans une certaine mesure, les déplacements ou la fixité des incidents, i.e. les comportements des agresseurs. Il est probable que cette dynamique explique les différents états de l'autocorrélation spatiale en criminologie. Mais notre objectif dans ce texte se limite à une simple présentation.

Après avoir considéré ces utilités et apports de l'analyse spatiale, il devient nécessaire de signaler les principaux moyens actuellement utilisés dans le cadre des analyses les plus connues en criminologie. Les recherches faites notamment par Lebeau (1987) et Stephenson (1980) sont particulièrement éclairantes à ce sujet. Nous décrivons ci-après la construction de la carte mentale, les mesures centrographiques et l'évaluation de l'autocorrélation spatiale.

\section{CARTE MENTALE}

La carte mentale est généralement constituée à partir des réponses fournies par un échantillon de sujets habitant la zone étudiée. Cet échantillon doit être évidemment constitué selon les règles de l'art, sous peine de 
biais. Toutefois, l'expérience semble suggérer qu'il est rarement nécessaire de suréchantillonner dans ce contexte.

On envoie ou on présente aux répondants une carte du territoire étudié. ainsi qu'un bref questionnaire. La qualité de la carte utilisée est de première importance. Elle doit être claire et facile à lire. Ainsi, on indique plus clairement les principales rues, certains lieux tels que centres commerciaux, églises, parcs, etc. On demande au sujet de signaler par une croix ou de hachurer sur la carte les endroits où, selon sa perception, le phénomène à l'étude se produit le plus souvent, ou encore les endroits qui lui inspirent un sentiment donné, par exemple la peur.

L'information ainsi recueillie peut être traitée par des méthodes centrographiques mais la simple sommation des réponses peut convenir dans bien des cas. On obtient ainsi une carte indiquant la répartition et les densités du fait étudié selon les perceptions des répondants.

\section{MESURES CENTROGRAPHIQUES}

Les principales mesures centrographiques utilisables sont le centre moyen (mean center), la distance-type (standard distance) et la vélocité. À partir de ces mesures on a pu développer d'autres indices (par exemple le coefficient de circularité), mais nous nous limiterons à ces trois informations.

Le centre moyen constitue certainement la mesure de base. Le calcul se fait comme suit. Après avoir dressé une cartographie ponctuelle représentant les lieux d'occurrence d'un phenomène (exemple cambriolage) on traite au jugé à travers le nuage de points deux axes orthogonaux d'orientation est-ouest $(\mathrm{X})$, nord-sud (Y). Leur point d'intersection peut se situer au centre apparent de la distribution. Ces axes servent de référence à la définition ou localisation de chaque point de l'ensemble, ce qui permettra d'énoncer le centre moyen. Cette moyenne est la paire de coordonnées $(\bar{X}, \bar{Y})$, où $\bar{X}=$ la moyenne des distances à l'axe $\mathrm{X}$ et $\bar{Y}=$ la moyenne des distances à l'axe $Y$.

Ceci définit en quelque sorte le site moyen d'un phénomène. Cette mesure est un peu rudimentaire et admet des réserves. Ainsi, dans le cas d'une répartition bimodale ou présentant une forme en $L$ elle serait pratiquement inutilisable.

Il est également possible d'énoncer la distance-type (standard distance) à l'intérieur de la distribution. On peut l'exprimer comme suit.

$$
\mathrm{D}=\mathrm{S}^{2} \mathrm{x} \text { et } \mathrm{S}^{2} \mathrm{y}
$$

où $S^{2} x$ et $S^{2} y$ sont les variances de longitude et de latitude calculées à partir des distances aux mêmes axes $\mathrm{X}$ et $\mathrm{Y}$. Cette mesure permet de comparer les densités de plusieurs distributions. 
La connaissance du centre moyen conduit à d'autres informations intéressantes, notamment au sujet du mouvement spatio-temporel d'une configuration représentant un ensemble de crimes. Stephenson (op. cit.) utilise dans ce cas la notion de vélocité (V). On pose :

$$
\mathrm{V}=\mathrm{S} / \mathrm{T}
$$

où $\mathrm{S}=$ la distance séparant les centres moyens entre deux moments (exemple en 1986 et en 1993)

$\mathbf{T}=$ l'intervalle de temps entre ces deux moments (dans l'exemple $\mathrm{T}=$ 7 ans).

Cet indice, avec quelques remaniements, peut exprimer la turbulence. Par exemple, si on fait équivaloir la turbulence au nombre de mouvements d'un phénomène d'une période à l'autre, on désigne en fait le momentum. C'est le produit du nombre d'événements par la vélocité.

\section{AUTOCORRÉLATION SPATIALE}

Nous avons mentionné antérieurement l'importance de l'autocorrélation spatiale. Lorsque la répartition géographique d'une variable (sa carte) suggère l'existence d'une structure spatiale, on peut être amené à vérifier statistiquement s'il existe une autocorrélation significative et à établir son type avant d'utiliser des tests paramétriques tels que la corrélation, la régression ou l'analyse de variance. Cette étude peut être entreprise pour deux raisons diamétralement opposées. D'une part, un chercheur peut vouloir prouver qu'il n'existe pas réllement d'autocorrélation afin de justifier l'emploi de techniques statistiques paramétriques. On peut également rechercher la preuve de l'existence d'une structure spatiale afin de mieux l'analyser.

Il existe actuellement plusieurs méthodes d'évaluation de l'autocorrélation spatiale. Ainsi, on peut mentionner les coefficients $C$ de Geary et I de Moran. La présentation in extenso de ces coefficients entraînerait des considérations techniques assez détaillées et un exposé d'une ampleur qui dépasse le cadre de cet article. Nous renvoyons le lecteur intéressé aux textes de Cliff et Ord (1981) ou de Legendre et Legendre (1984a) (Costanzo, Halperi et Gale, op. cit.). Sur ce sujet, on doit suggérer vivement le livre de Griffith (1987).

On aura deviné que le calcul des indices et coefficients mentionnés dans ce texte implique des opérations laborieuses de cartographie, la manipulation d'une masse de données parfois considérable et suggère des coûts probibitifs. Mais le développement en informatique de nouveaux progiciels amène de nouvelles possibilités et un allégement considérable du travail 
requis (voir MAPINFO et STAC, i.e. Spatial and Temporal Analysis of Crime utilisé à Chicago).

Nous avons évoqué succinctement dans ces pages les moyens et méthodes qui peuvent être employés dans l'analyse spatiale en criminologie. Nous soumettons ci-après pour fin d'illustration deux exemples d'application tirés de nos travaux. Il s'agit d'une utilisation de la carte mentale et de l'évaluation de l'autocorrélation spatiale.

Le premier exemple est une étude de la peur du crime dans le quartier Côte-des-Neiges, à Montréal, au moyen de la carte mentale. Notre instrument était un plan simplifié du quartier accompagné de neuf questions dont huit s'adressaient aux caractéristiques des répondants. La question principale visait à détecter les zones perçues comme risquées.

Elle était formulée comme suit :

"Hachurez (IIII), sur la carte, les endroits ou les bouts de rue que vous trouvez, la nuit par exemple, moins sûrs ou plus risqués. Si ce sont des places précises, un immeuble par exemple, marquez-les d'une croix (X). »

Le questionnaire était rédigé dans les deux langues officielles et expédié à 400 répondants choisis sur une base aléatoire à partir des listes électorales. Nous évacuons la présentation des nombreux résultats obtenus qui ne semblent pas pertinents dans ce texte et soumettons ci-après la carte synthèse (fig. 1).

Cette carte a été obtenue par simple sommation des réponses. Ainsi, lorsque plusieurs répondants signalent une même zone ou rue, celle-ci devient facilement repérable. On découvre de cette façon ce qu'on a appelé une «surface invisible du stress» ou une topographie de la peur. Les zones de haute densité du stress se situaient autour du centre commercial de Côte-des-Neiges, notamment sur les rues Barclay, Bedford et Goyer.

On est naturellement amené à se demander ce qui caractérise ces aires anxiogènes, ce qui conduit la majorité des répondants à les situer hors de la civilisation. Parmi les autres informations colligées on a pu constater qu'on n'y signalait pas un fort taux de criminalité. Par contre, les endroits désignés étaient plutôt délabrés et abritaient une population relativement « marginale », comprendre par cela des chômeurs, des assistés sociaux et surtout des immigrants asiatiques ou de race noire. On découvrait à nouveau que la peur du crime est une des composantes ou soutien de la xénophobie.

Le deuxième exemple est une évaluation de l'autocorrélation spatiale. Pour les fins de cette étude, nous avions choisi d'analyser la répartition et l'évolution spatiale des vols qualifiés et des cambriolages.

Les données de base provenaient de la police de la Communauté urbaine de Montréal (SPCUM). Il s'agissait en fait de la liste complète de 
toutes les plaintes logées auprès de la police et des rapports d'événements d'un district administratif, avec la date et l'adresse exacte de l'incident. Le territoire choisi était celui du district de police $n^{0} 33$, qui représente une zone sensible du centre-ville de Montréal. La période d'observation s'étendait du $1^{\text {er }}$ janvier au 31 décembre de l'année 1989.

L'analyse de la structure spatiale a été réalisée selon trois méthodes différentes, à savoir :

a) l'utilisation des coefficients d'autocorrélation spatiale (I de Moran et C de Geary) et des corrélogrammes associés ;

b) un regroupement spatial, i.e. nous avons opéré un regroupement des zones étudiées sous contrainte de contiguïté spatiale, afin de vérifier la configuration la plus probable de la structure en termes de groupes homogènes; en d'autres termes, nous cherchions à mettre en évidence des plaques de densité analogue pour chacun des délits; la méthode utilisée est en fait une variante de l'analyse en grappe (cluster analysis) ;

c) une analyse des surfaces théoriques (trend surface analysis). Cette méthode permet d'exprimer les variations d'une variable simple, en différents points de l'espace, comme une fonction des coordonnées spatiales des points d'échantillonnage :

$$
\mathrm{z}=f(\mathrm{x}, \mathrm{y})
$$

où $\mathrm{z}$ représente les valeurs de la variable dépendante $\mathrm{z}$ prédites par la fonction, alors que $x$ et y représentent les valeurs des coordonnées spatiales des points d'échantillonnage dans un plan cartésien. Une fonction du premier degré $x=b_{0}+b_{1} x+b_{2} y$, qui est un modèle linéaire, permet de faire passer un plan à travers les différentes valeurs de z sur la carte.

Les résultats détaillés de ces méthodes et analyses se trouvent dans un article de Élie et Legendre (1992).

La prẹière méthode a produit des résuiltats incertains et difficilement interprétables dans le contexte. Par contre. les deux autres approches ont nettement fait ressortir la structure spatiale et l'autocorrélation. Nous soumettons, seulement pour illustration (fig. 2), les valeurs prédites par le modele spatial de régression pour les vols par effraction. La carte montre d'abord l'existence d'un gradient linéaire d'est en ouest avec maximum à l'ouest; du nord au sud, la structure est plus complexe: elle crô̂t depuis le secteur de la rue Sherbrooke pour atteindre son maximum sur la ligne reliant les stations de métro Place-des-Arts, Saint-Laurent, Berri-UQAM, Beaudry et Papineau, et s'abaisser de nouveau vers le fleuve. Les valeurs maximales d'introduction par effraction prédites par ce modèle spatial se situent donc autour de la station de métro Place-des-Arts. 
Figure 1

Distribution de la peur du crime dans un quartier de Montréal

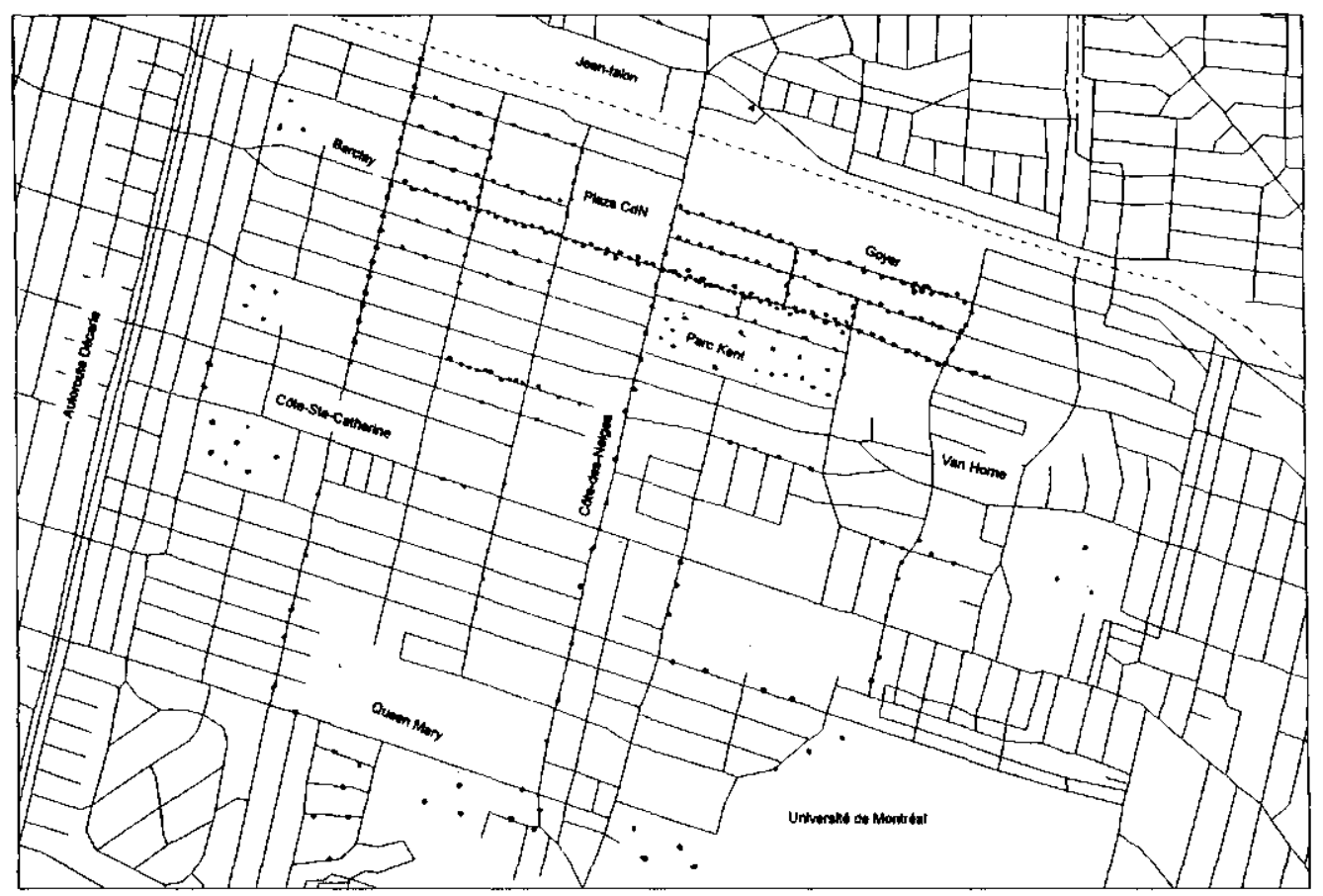


Figure 2

La structure spatiale du vol par effraction dans un quartier de Montréal

\section{Nord}

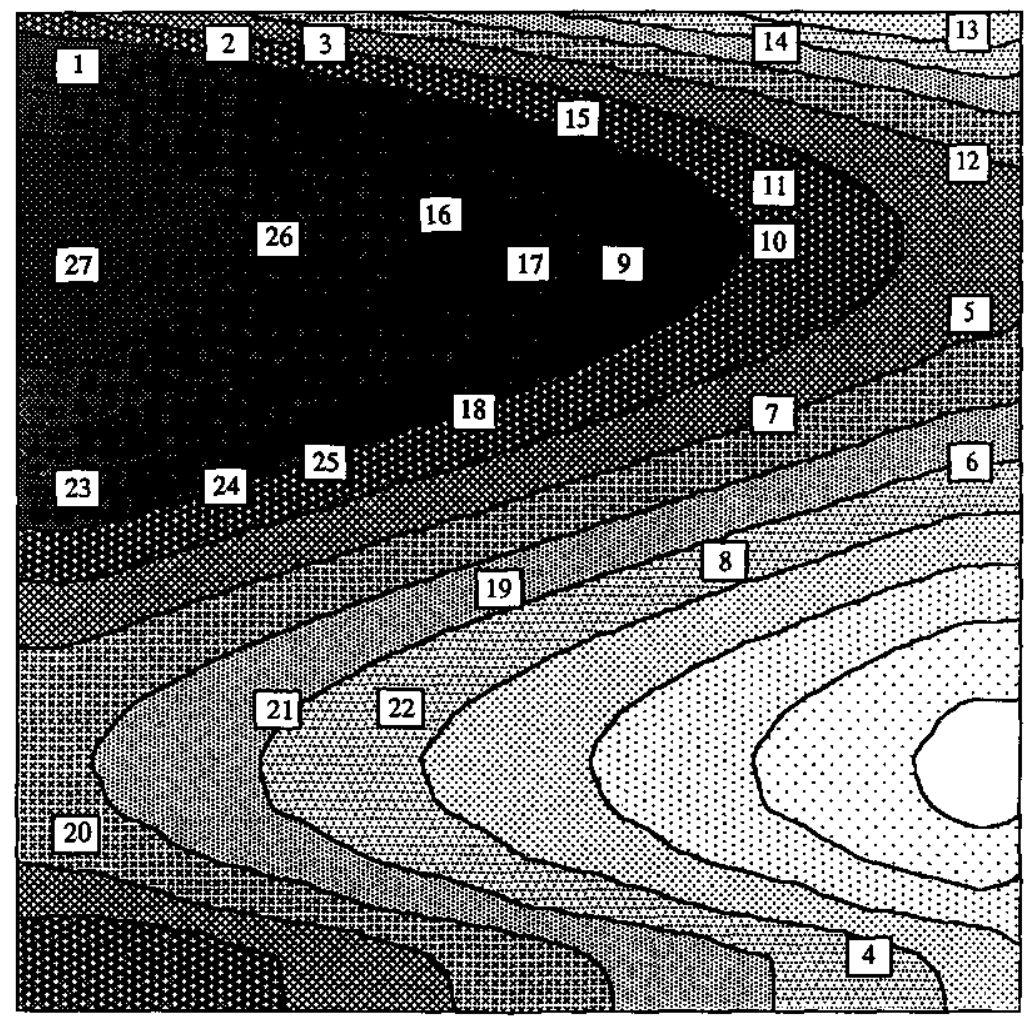

Sud 


\section{CONCLUSION}

Après ces esquisses des utilités, moyens et méthodes de l'analyse spatiale, il semble opportun, en guise de conclusion, de considérer quelques notions et concepts importants qui sont susceptibles de rendre cette approche fertile.

À ce sujet, Brantingham et Jeffery (1981) ont rappelé, avec pertinence, des aspects négligés des propositions théoriques élaborées autour de l'école de Chicago, notamment celles de Ernest W. Burgess. Le point est important parce que la question que nous devons poser encore maintenant, étant donné les nouveaux moyens disponibles, concerne la possibilité et le mode de passage de l'analyse spatiale à une véritable écologie de la criminalité. Dans cette perspective, la reconsidération de certaines notions peut s'avérer payante pour l'avancement d'une partie des théories criminologiques.

Selon ces auteurs, les implications et la richesse de l'approche écologique auraient été sous-utilisées et mésusées, le meilleur exemple en ce sens étant l'œuvre de Burgess.

Celui-ci établissait, en 1925 déjà, le rôle des situations géographiques, des distances et de la mobilité dans l'avènement de certains crimes. Sa théorie du développement urbain et de la criminalité associée retenait trois processus centraux qu'on peut résumer comme suit.

Il signalait d'abord la dynamique de l'«expansion » à partir d'un centre économique. Il voyait cette expansion comme étant (nécessairement) combinée à un autre fait qu'il a désigné par le terme de «métabolisme», i.e. un processus de désorganisation et de réorganisation au cours duquel la cité assimilerait nouveautés et immigrants. Enfin, il retenait le fait de la «mobilité ", i.e. les déplacements et communications, englobant ainsi dans sa position théorique l'importance des communications et des mouvements dans l'élaboration des formes urbaines.

Nous rejoignons ces auteurs en constatant que ces concepts, associés aux moyens actuels de l'analyse spatiale, peuvent constituer une voie de renouveau. Nous sommes toutefois tenté de suggérer, comme ajout, l'observation de deux états contrastés, et mesurables, de la criminalité sur un territoire. Le premier serait la viscosité, i.e. la permanence des incidents dans une zone et leur forte connexité spatiale (densité). Ceci peut être évalué (autocorrélation, distance-type) et suggère des théories de portée moyenne, à teinte déterministe. Le second serait la turbulence, caractérisée par la fluidité et la mobilité des événements, par l'entropie apparente et l'accroissement de la complexité. Il appelle des théories plus formelles et de portée générale. 


\section{BIBLIOGRAPHIE}

BALDWIN, J. (1975), "British Areat Studies : An Assessment », The British Journal of Criminology.

BRANTINGHAM, P.J. et JEFFERY, R. (1981), "Crime, Space and Criminological Theory ", in Environmental Criminology, Brantingham, P.J. el Brantingham, P.L. (éd.) (1981), Sage Publications.

BRANTINGHAM, P.J. et BRANTINGHAM, P.L. (1981), Environmental Criminology, Sage Publications.

BURGESS, E.W. (1925a), «The Growth of the City", in R.E. Park, E.W. Burgess et M. McKenzie (éd.), The City, Chicago, University of Chicago Press.

BYRNE, J.M. et SAMSON, R.J. (1986), The social Ecology of Crime. New York, Springer-Verlag.

CLIFF, A.D. et ORD, J.K. (1981), Spatial Processes : Models and Application, Pion Limited, London.

Communities and Crime (1986), in REISS, A.J. Jr. et TONRY, M. (ed.), The University of Chicago Press.

COSTANZO, M.C., HALPERIN, W.C. el GALE, N. (1986), "Criminal Mobility and the Directional Component in Journeys to Crime", in Motropolitan Crime Patterns, éd. Figlio, R.M., Hakim, S. et Rengert, G.F., New York, Criminal Justice Press.

ÉLIE, D. et THÉORÊT, B. (1984), Les analyses spatiales de la criminalité. Partie I. Inventaire des études et tentative de bilan (1832-1980), rapport de recherche non publié.

ELIE, D. et LEGENDRE, P. (1992), «Autocorrélation spatiale et déplacement de la criminalite $n$, Criminologie, vol. XXV, $\mathrm{n}^{0} 2$.

FIGLIO, R.M., HAKIN, S. et RENGERT, G.F. (1986), Metropolitan Crime Patterns. Kennendale TX, Criminal Justice Press.

GEORGES-ABEYIE, D. el HARRIES, K.D. (1980), Crime: A spatial perspective. New York, Columbia University Press.

GRIFFITH, D.A. (1987), Spatial Autocorrélation. A primer, Association of American Geographers.

GUERRY. A.M. (1833), Essai sur la statistigue morale de la France, Paris, Crochard.

HARRIES, K.D. (1974), The Geography of Crime and Justice, MeGraw-Hill Ine.

LEBEAU, J.L. (1987), «The Methods and Measures of Centrography and the Spatial Dynamics of Rape », Journal of Quantitative Criminology, vol. 3, $\mathrm{n}^{\circ} 2$.

LEGENDRE, L. et LEGENDRE, P. (1984a), Écologie numérique, tome 2 : La structure des données ćcologiques ( $2^{\mathrm{e}}$ édition), Masson, Paris et Les Presses de l'Université du Québec.

MALTZ, M.D., GORDON, A.C., FRIEDMAN, W. (1991), Mapping Crime in Its Community Setting. Event Geography Analysis. Ed. Springer-Verlag, New York Inc.

MAYHEW, H. (1862), London Labour and the London Poor, Dover Publication; New York. Metropolitan Crime Patterns (1986), In FIGLIO, R.M., HAKIM, S. et RENGERT, G.F. (éd.) Criminal Justice Press. 
PYLE, G.F. (1980), « Systematic Sociospatial Variation in Perceptions of Crime Location and Severity», in Crime. A Spatial Perspective, éd. Georges-Abeyie, D. et Harris, K.D., Columbia University Press.

SHAW, C.R. et MCKAY, H.D. (1931), Social Factors in Juvenile Delinquency, Washington, D.C., Government Printing Office.

SHERMAN, W.L., GARTIN, P.R., BUERGER, M.E. (1989), Criminology, vol. 27, $\mathrm{n}^{\mathrm{a}} 1$.

STEPHENSON, L.K., "Centrographic Analysis of Crime», in Crime. A Spatial Perspective (1980), Georges-Abeyie, D.E. et Harris, K.D. (éd.), Columbia University Press.

The Social Ecology of Crime (1986), in BYRNE, J.M. et SAMPSON, R.J. (Ed.), Springer-Verlag, New York Inc.

TREMBLAY, P., CORDEAU, G., KACZOROWSKI, J. (1993), «Cartes mentales, écologie criminelle et sentiment d'insécurité des femmes en milieu urbain : une enquête pilote ", Revue canadienne de criminologie, vol. $35, \mathrm{n}^{\circ} 1$. 